\title{
The Longer Term Evolution of Magnetic Field and Mass Flow in a Decaying Active Region
}

\author{
Hongqi Zhang ${ }^{1}$, Guoxiang $\mathrm{Ai}^{1}$, Haimin Wang ${ }^{2}$, \\ Harold Zirin ${ }^{2}$, Alan Patterson ${ }^{2}$ \\ ${ }^{1}$ Beijing Astronomical Observatory, Chinese Academy of Sciences, P. R. \\ China \\ ${ }^{2}$ Big Bear Solar Observatory, California Institute of Technology, U.S.A.
}

During September 24-29, 1987, the longitudinal magnetic fields of a decaying active region (AR 4855) were observed almost continuously at Big Bear and Huairou Solar Observatories. The target consisted of an $\alpha p$ sunspot and surrounding enhanced network. We achieved a 75-hour coverage with 4 night-time gaps of six to eight hours. The images were then combined to made a continuous movie.

The BBSO Videomagnetograph (VMG) System was originally developed by Leighton and Smithson and has been improved in recent years (Zirin, 1985). This system has made it possible to study very weak photospheric magnetic structures.

The vector videomagnetograph system at Huairou Solar Observing Station of Beijing Astronomical Observatory, located on an island of the Huairou reservoir $60 \mathrm{~km} \mathrm{NE}$ of Beijing, was designed by G. Ai (1987). It consists of a $35 \mathrm{~cm}$ vacuum telescope, a $1 / 8 \AA$ birefringent filter with 3 sets of KD*P crystal modulators, a CCD camera and an Imaging Technology 151 system controlled by an AST386 system, which transmits the data to a Vax/11-750. It works at either of two spectral lines: FeI $\lambda 5324.19 \AA$ for photospheric magnetic and line-of-sight velocity observations and $\mathrm{H} \beta \lambda 4861.34 \AA$ for chromospheric observation. The field of view is about $5.7^{\prime} \times 4^{\prime}$ and the pixel resolution is about $0.7^{\prime \prime} \times 0.4^{\prime \prime}$. This videomagnetograph also has high spatial and temporal resolution. With good seeing conditions, the smallest magnetic features visible are smaller than $2^{\prime \prime}$. The longitudinal component (Stokes parameter V) of the vector magnetic field in the solar photosphere was measured at $0.075 \AA$ from the line center of $\mathrm{FeI} \lambda 5324.19 \AA$ and the transverse components (Stokes parameters $\mathrm{Q}$ and $\mathrm{U}$ ) at the line center. The photospheric Dopplergrams were measured with the subtraction technique at $\pm 0.15 \AA$ from the line center of $\mathrm{FeI} \lambda 5324.19 \AA$ and the chromospheric Dopplergrams at $\pm 0.24 \AA$ from the center of $\mathrm{H} \beta \lambda 4861.34 \AA$. After performing a $4 \times 3$ pixel $\left(2^{\prime \prime} \times 2.1^{\prime \prime}\right)$ spatial averaging and integrating 255 frames, the signal to noise ratio is 5600 corresponding to $\pm 2 \mathrm{G}$ of longitudinal field, $\pm 15 \mathrm{G}$ of transverse field, $\pm 2 \mathrm{~m} / \mathrm{s}$ lineof-sight velocity field for the photosphere and $\pm 8 \mathrm{~m} / \mathrm{s}$ for the chromosphere. 
The decaying active region was located on the northern hemisphere of the Sun and the region was west of CMP. The sunspot had negative magnetic polarity. Some positive elements occurred on the west penumbra of the sunspot, which was in the opposite direction from the solar disk center. The transverse components of the sunspots magnetic field showed the spoke-like extensions from the center and connected the longitudinal fields of opposite polarity. We showed that the positive structures intruded gradually into the penumbra of the spot from September 25 to September 27 and the heliocentric angle of the target increased from about 30 to 60 . The transverse components of fields in the penumbra were strong. From the distributions of the vector magnetic fields, we could infer that the longitudinal magnetic structures of opposite polarity that intrude into the sunspot were probably the projection effects of inclined magnetic force lines from a sunspot, that returned to the solar surface near to or in the penumbra, and the lines of force were almost parallel to the solar surface.

The outward flow of magnetic flux plays a very important role in the evolutionary history of a decaying sunspot. The shift and velocity pattern of the magnetic elements is shown in Fig. 1. The magnetic fields consist of the moving magnetic features (MMF) flowing outward around the sunspot. In the westward direction this flow is almost exclusively monopolar and opposite in sign to the umbral field, even though part of the moving magnetic features have mixed magnetic polarities (predominantly of positive polarity with some very weaker negative elements). However we found that some of negative magnetic fragments of the sunspot were moving outwards from the edge of the magnetic field of the sunspot at an average speed of about $0.3 \mathrm{~km} / \mathrm{s}$. The direction of these motions appeared irregular. Some of fragments formed a part of network magnetic fields of the same magnetic polarities. Some moved along the outer side of the moat and approached and cancelled with magnetic elements of opposite sign.

The decay rate of sunspot magnetic flux for the same polarity as the associated sunspot is found to be $10^{20} \mathrm{Mx} / \mathrm{h}$ on September 25 and $4 \times 10^{19} \mathrm{Mx} / \mathrm{h}$ on September 26 , but for the opposite polarity $-10^{18} \mathrm{Mx} / \mathrm{h}$ on September 25 and $0.8 \times 10^{17} \mathrm{Mx} / \mathrm{h}$ on September 26. The increase of the sunspot magnetic flux of the opposite polarity on September 25 is due to a projection effect associated with the rotation of the sunspot, even through the magnetic flux with polarity opposite to parent sunspot move out from the sunspot. The high-quality movies show these larger magnetic fragments to be eroding the sunspot edge. The fragments of magnetic fields outflowing from the sunspot probably are the main mechanism of magnetic flux loss of the sunspot.

The motions of the magnetic elements of the enhanced network also appear irregular, the average velocity of elements is about $0.3 \mathrm{~km} / \mathrm{s}$. We find that some of magnetic elements converge and merge. Some magnetic elements break and the fragments of the magnetic field move away in different directions, other elements suddenly change direction and others still move consistently in one direction. Some magnetic elements move about $3.7 \times 10^{4} \mathrm{~km}$ at 43 hours whereas some, of opposite polarities, collide, in which case the total flux then decreases because of cancellation. 


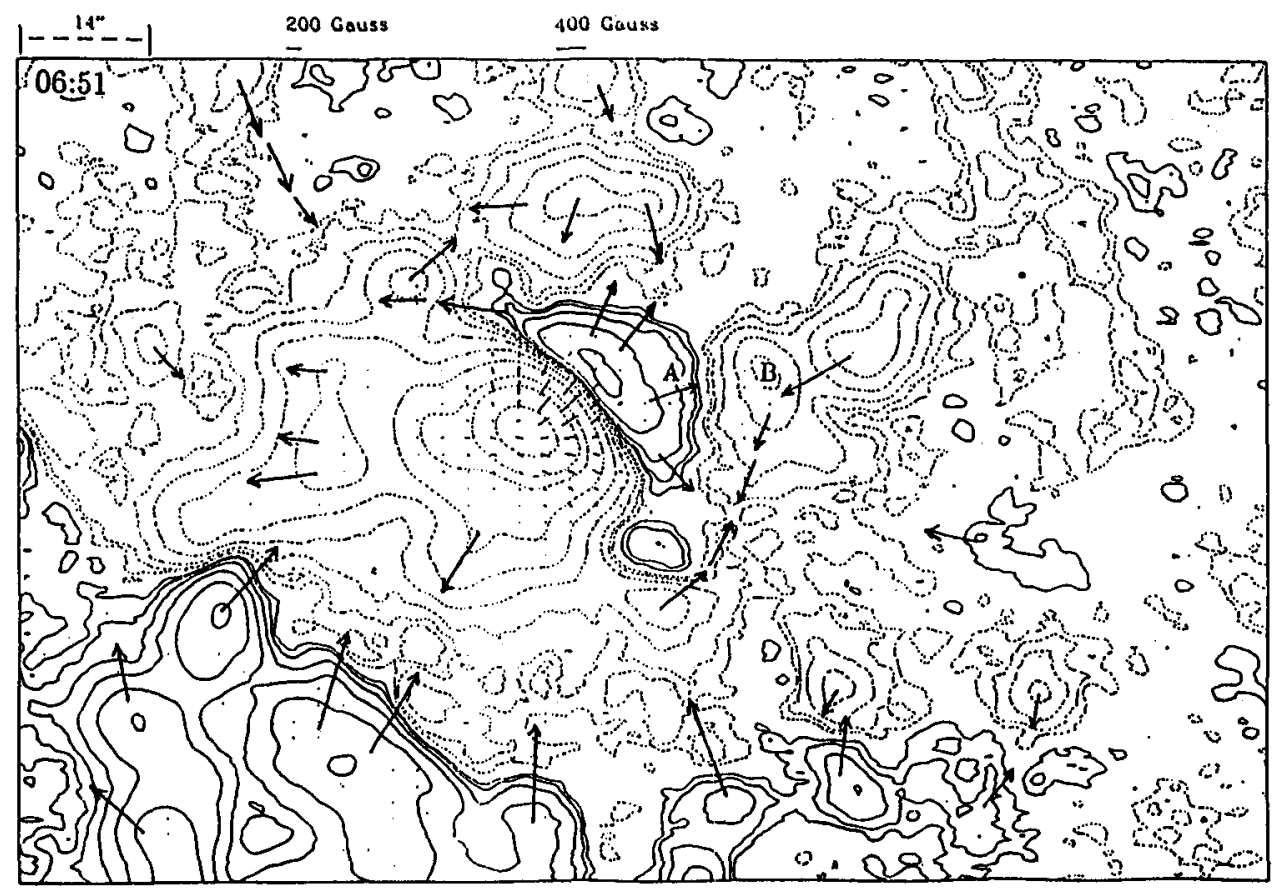

Fig. 1. The vector magnetogram of Active Region 4855 of Sept. 26, 1987. The arrows show the shift and direction of motion of the magnetic features averaged over September 26 and 27. Solid (dashed) contours correspond to positive (negative) fields of 5, 20, 40, $80,160,320,640,960$ Gauss. North is at the top, west is at right.

We now present the results of attempting to measure the velocity of MMF's and velocity fields simultaneously on Sept. 26 and 27 . The profiles of velocity and magnetic fields are asymmetric through the sunspot. The distributions of velocity and magnetic fields are different. By tracking individual features, we found that the speed of moving magnetic features is about $1.0-2.5 \mathrm{~km} / \mathrm{s}$, which is greater than photospheric Doppler measurement. On the other hand, the negative fragments of sunspot field move outward from the edge of sunspot at an average speed of about $0.3 \mathrm{~km} / \mathrm{s}$, similar to the corresponding Doppler velocity. We can infer that the mechanism driving the motion of the magnetic fragments may be different from that of discussed above for the moving magnetic features. It probably is the outward motions of the feet of the magnetic lines of force, near to the outer edge of the photospheric penumbra.

On September 26 and 27, our observed chromospheric Doppler velocities from this decaying active region mainly reflect components of the horizontal velocities. We compared the Doppler measurements of photosphere and chromosphere. The immediate result shown is that the chromospheric and photospheric velocities are generally in opposite phase, i.e. if the chromosphere has a red shift, the photosphere 
has a blue shift. Although the maximum of the chromospheric field has a small offset from the minimum of the photospheric field on Sept. 26, the maximum and the minimum coincide on Sept. 27. This means that the direction of the chromospheric mass motion in the superpenumbra is opposite to that of the photosphere. We find that the maximum of the chromospheric Doppler velocity fields shifts toward the penumbra from outside of the sunspot from September 26 to 27. The velocity maximum occurs to the outside of the inversion of longitudinal magnetic fields on the penumbra. Comparing the distribution of the chromospheric Doppler velocity fields with the photospheric vector magnetograms, it is difficult to infer the result that the directions of chromospheric mass motion are completely parallel to the directions of chromospheric force lines above our observed photospheric vector magnetic fields, especially near the inversion line of the photospheric longitudinal magnetic fields. It is possible that the magnetic lines of force are curved in the chromosphere or that mass flows along a complex return circuit in the sunspot atmosphere.

Acknowledgements. We are grateful to the staff at Huairou Solar Observing Station and Big Bear Solar Observatory for their support in making the observations. This research was supported by the Chinese Academy of Sciences and National Science Foundation of China and NSF of U.S.A. under the grant NSF INT-8814395.

\section{References}

Ai, G.: 1987, Publications of Beijing Astronomical Observatory 9,27

Zirin, H.: 1986, Aust. J. Phys. 38, 961 